\title{
Measurement of Unsteady Pressure Data on a Large HSCT Semispan Wing and Comparison with Analysis
}

\author{
Robert C. Scott, ${ }^{*}$ Walter A. Silva, ${ }^{\dagger}$ James R. Florance, ${ }^{\ddagger}$ and Donald F. Keller $^{\S}$ \\ Aeroelasticity Branch, NASA Langley Research Center, Hampton, VA 23681
}

\begin{abstract}
Experimental data from wind-tunnel tests of the Rigid Semispan Model (RSM) performed at NASA Langley's Transonic Dynamics Tunnel (TDT) are presented. The primary focus of the paper is on data obtained from testing of the RSM on the Oscillating Turntable (OTT). The OTT is capable of oscillating models in pitch at various amplitudes and frequencies about mean angles of attack. Steady and unsteady pressure data obtained during testing of the RSM on the OTT is presented and compared to data obtained from previous tests of the RSM on a load balance and on a Pitch and Plunge Apparatus (PAPA). Testing of the RSM on the PAPA resulted in flutter boundaries that were strongly dependent on angle of attack across the Mach number range. Pressure data from all three tests indicates the existence of vortical flows at moderate angles of attack. The correlation between the vortical flows and the unusual flutter boundaries from the RSM/PAPA test is discussed. Comparisons of experimental data with analyses using the CFL3Dv6 computational fluid dynamics code are presented.
\end{abstract}

\section{Introduction}

A primary goal of the Transonic Dynamics Tunnel (TDT) at the NAS $\Lambda$ Langley Research Center (LaRC) is to acquire high-quality wind-tunnel data for the validation of aeroelastic analysis methods including the development and application of aeroelastic computational fluid dynamics (CFD) codes. The need for such data has been recognized for many years and has led to the development of various programs to generate a database of high quality unsteady data. The RTO effort ${ }^{1}$ is an example of an international cooperative effort to define, compile, and disseminate highquality experimental data sets. $\Lambda \mathrm{N} \Lambda \mathrm{S} \Lambda$ Langley effort was the Benchmark Models Program (BMP). The BMP resulted in the testing of several configurations for which steady and unsteady pressures and flutter data were obtained. ${ }^{2-6}$ Testing of the Benchmark $\Lambda$ ctive Controls Technology (B $\Lambda \mathrm{CT}$ ) model ${ }^{7}$ comprises the most recent data set for this class of wind-tunnel models.

The Rigid Semispan Model (RSM) which is the focus of this paper and an identically shaped flexible version ${ }^{8}$ of the RSM were defined near the end of the NASA LaRC's BMP. These wind-tunnel models, intended to be representative of a high-speed civil transport (HSCT), became part of the Aeroelasticity

\footnotetext{
*Aerospace Engineer, AIAA Senior Member.

†Senior Research Scientist, AIAA Senior Member.

† Aerospace Engineer, AIAA Senior Member.

$\S$ Aerospace Engineer

Copyright (C) 2002 by the American Institute of Aeronautics and Astronautics, Inc. No copyright is asserted in the United States under Title 17 , U.S. Code. The U.S. Government has a royaltyfree license to exercise all rights under the copyright claimed herein for Governmental Purposes. All other rights are reserved by the copyright owner.
}

element of the High Speed Research (HSR) program.

The objective of the Aeroelasticity element of the HSR program was to provide validated analyses, design tools, and demonstrate technology readiness to accurately predict and solve the aeroelastic problems of an advanced high-speed civil transport (HSCT). $\Lambda$ s part of this task, a wind-tunnel models subtask was created to measure and document the aeroelastic characteristics, the steady and unsteady pressures and forces, and the aeroelastic stability boundaries for models of increasing complexity. One of the goals of this activity was to perform various tests on the RSM.

The RSM was tested on three different mount systems. These were a 5 degree of freedom (DOF) bal-

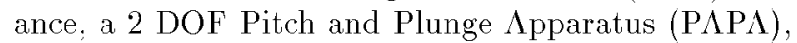
and an Oscillating Turntable (OTT). The purpose of this paper is to describe the OTT database, and present samples of steady and unsteady pressure data. Where applicable, comparisons will be made between OTT data and data acquired on previous wind-tunnel tests. $\Lambda$ dditionally, $\mathrm{CFD}$ results will be compared with experimental data. Discussion of the data and results will demonstrate that the RSM database provides significant challenges for CFD codes.

\section{Experimental Apparatus}

\section{Transonic Dynamics Tunnel}

The Langley Transonic Dynamics Tunnel (TDT) is a unique national facility dedicated to identifying: understanding, and solving relevant aeroelastic and aeroservoelastic problems. The TDT is a closedcircuit, continuous-flow, variable-pressure, wind tunnel with a 16-foot square test section with cropped corners. The tunnel uses either air or a heavy gas as 
the test medium and can operate at stagnation pressures from near vacuum to atmospheric, has a Mach number range from near zero to 1.2 and is capable of maximum Reynolds numbers of about 3 million per foot in air and 10 million per foot in heavy gas. Until 1996, the TDT used dichlorodifluoromethane, R-12, as the heavy gas test medium; since then the TDT has used $1,1,1,2$ tetrafluoroethane, $\mathrm{R}-134 \mathrm{a},{ }^{9}, 10$ an environmentally acceptable alternative to $\mathrm{R}-12$.

The TDT is specially configured for flutter testing, with excellent model visibility from the control room and a rapid tunnel shutdown capability for model safety. Model mount systems include a sidewall turntable for semispan models, a variety of stings for full-span models, and a cable-mount system for "flying" models. The TDT also offers an airstream oscillation system for gust studies as well as digital controllers and supporting systems for active controls testing. Testing in heavy gas has important advantages over testing in air: improved model to full-scale similitude (which results in heavier, easier to build models with lower model elastic mode frequencies): higher Reynolds numbers, and reduced tunnel power requirements. These capabilities make the TDT one of the best-suited facilities in the world for flutter testing large, aeroelastically-scaled models at transonic speeds.

\section{Rigid Semispan Model (RSM)}

The RSM planform is a $1 / 12$ th scale configuration based on an early design known as the Reference $\mathrm{H}$ configuration. Model airfoil shapes were based on those of the Reference $\mathrm{H}$, with the model wing thickness being increased to a constant $4 \%$ thickness-tochord ratio in order to accommodate pressure instrumentation at the wing tip. The model was designed to be very stiff to allow the measurement of aerodynamic properties without the effects of structural deformations.

Figure 1 shows the planform layout and main components of the RSM including the three primary mounts used during the various wind-tunnel tests. The leading and trailing edges were removable in order to access pressure instrumentation in those regions. $\Lambda$ removable tip cap allowed access to pressure instrumentation at the wing tip. The RSM could be tested with and without a pair of flow-through nacelles. The nacelles were rigidly attached to pylons on the lower, inboard surface of the wing. The RSM wing had a graphite epoxy composite structure with an open-cell foam core. The RSM was re-built in 1995 after experiencing a failure of the bond of the upper and lower surfaces. Rivets were inserted along the front and rear spars to eliminate the possibility of a similar failure in future testing, and the original four-pound (i.e. a density of $4 \mathrm{lb} / \mathrm{ft}^{3}$ ) foam core was replaced with an eight-pound foam core for added strength and dura-



Fig. 1 Planform, model details, and instrumentation layout for the RSM wind-tunnel model.

bility.

The RSM was tested with a rigid fuselage fairing which displaced the model away from the wind-tunnel wall boundary layer while serving as an aerodynamic boundary condition at the wing root. Additionally, the rigid fuselage fairing provided an aerodynamic shield for the hardware, instrumentation, and wire bundles located at the wing root. Three different fuselage fairings were used with the RSM. The lengths of these fuselage fairings were approximately 18, 14, and 11 feet. The 18 and 11-foot fuselages had a near rectangular cross-section with rounded corners while the 14-foot fuselage was approximately semi-circular. The aft ends of the 18 and 11-foot fuselages were rather blunt, while the 14 -foot fuselage extended further downstream with a more gradual taper to reduce turbulence near the trailing edge of the wing. The center of rotation for the 18 and 11-foot fuselages was 142 inches aft of a reference point defined by the leading edge of the 18 -foot fuselage. The center of rotation for the 14-foot fuselage was 133 inches aft of this point. This resulted in the wing center of rotation being moved 9 inches forward when installed on the 14-foot fuselage. $\Lambda$ photograph of the RSM, engine nacelles, and the 18 -foot long fuselage fairing installed in the TDT test section is shown in figure 2 .

The instrumentation layout for the RSM (visible in figure 1) consisted of 131 insitu unsteady pressure transducers located at the 10, 30, 60, and $95 \%$ span stations. Six additional unsteady pressure transducers were installed at the $20 \%$ chord station for the 20 , 45 , and $75 \%$ span stations for both upper and lower surfaces. Channels were carved into the foam core to accommodate the wiring for the instrumentation. Instrumentation also included accelerometers installed throughout the wing. The 18 and 11 -foot long fuselage fairings were instrumented with 120 steady pressure orifices at seven fuselage stations. The 14-foot long fuselage fairing was instrumented with unsteady pressure transducers.

$\Lambda$ remotely commanded, hydraulically actuated control surface was available on the RSM. This flap was capable of rotating \pm 15 degrees without the engine 


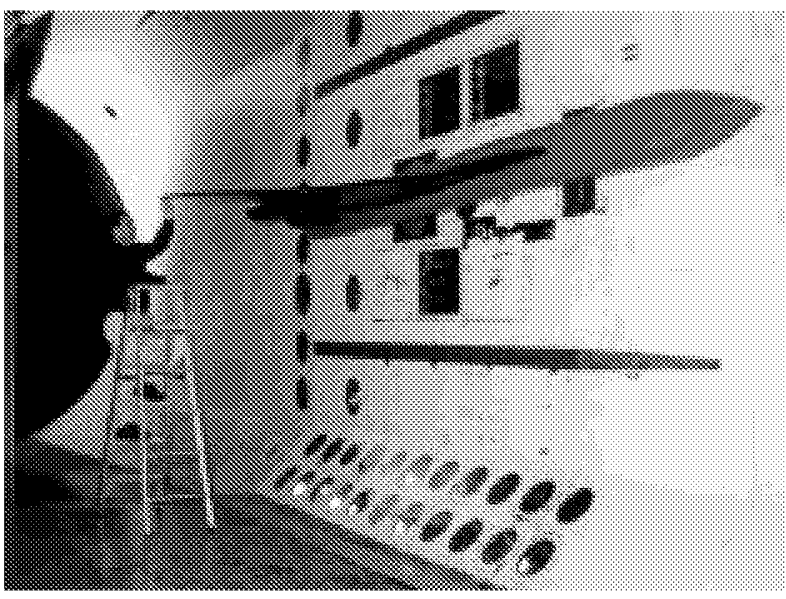

Fig. 2 The RSM and 18 foot fuselage mounted in the TDT test section.

nacelles installed. When installed, the inboard engine nacelle inhibited downward motion of the trailing-edge flap. The flap was used to obtain unsteady aerodynamic data on the balance and $\mathrm{P} \Lambda \mathrm{P} \Lambda$ mount systems.

\section{Balance}

$\Lambda$ five-degree-of-freedom sidewall balance was used for the measurement of loads on the RSM. The loads measured were for the wing alone and not for the combined wing/fuselage fairing configuration. Measurement of the combined wing/fuselage fairing loads would have impeded computational validation efforts since the quality of the data would have been compromised by the complex interaction of the fuselage with the tunnel wall boundary layer. In addition, loads data from a combined wing/fuselage fairing configuration is of questionable value since it is impossible to discern the contribution of each component (wing or fuselage) from the measured load, again, impeding validation efforts. For these reasons, the wing was attached to the balance but the fuselage fairing was not. The wing/balance system and the fuselage fairing were attached to the tunnel turntable via independent hardware connections.

During one RSM wind-tunnel test a strut was used in place of the balance. With the exception of wing loads, the same types of data were acquired on the strut as on the balance. In addition, the load limits for the strut are higher than for the balance, and thus, the strut allowed data to be acquired at higher angles of attack than was possible on the balance.

\section{Pitch And Plunge Apparatus (PAPA)}

The RSM PAPA mount is an updated version of the PAPA mount used in NASA Langley's Benchmark Models (BMP) program. ${ }^{2,4-7}$ The BMP P $\Lambda \mathrm{P} \Lambda$ mount ${ }^{11}$ was developed at $N \Lambda S \Lambda$ Langley Research Center, and when used with a rigid model, provides the two flexible degrees of freedom (pitch and plunge) needed for classical flutter. The RSM PAPA is much

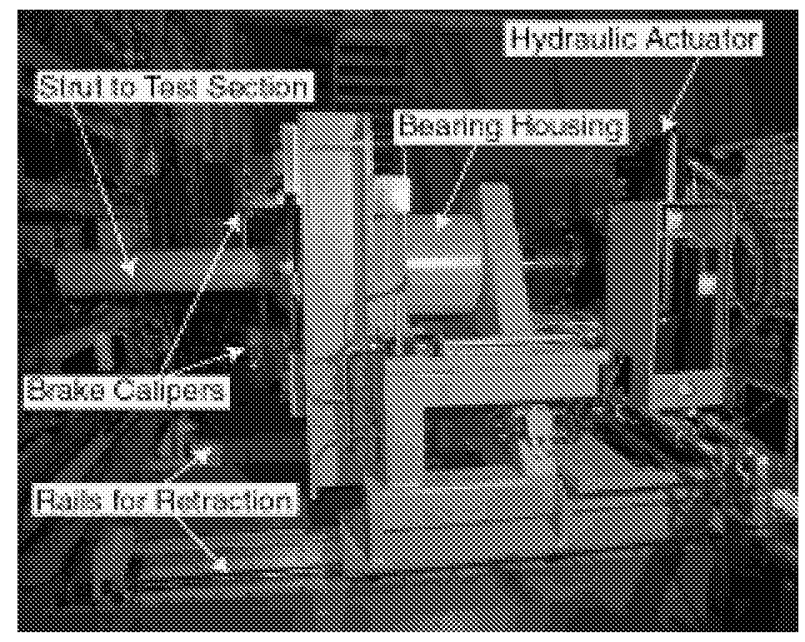

Fig. 3 Side view of the Oscillating Turntable installed in the TDT.

stiffer than the BMP P $\Lambda \mathrm{P} \Lambda$ so it can accommodate the increased mass, pitch inertia, and aerodynamic forces and moments of the RSM as compared to the BMP models. The BMP PAP $\Lambda$ consisted of 4 rods between a fixed and a moving plate, while the RSM $\mathrm{P} \Lambda \mathrm{P} \Lambda$ had 8 rods. The rigid-body plunge mode consists of vertical translation of the wing model and the rigid-body pitch mode consists of rotation of the wing model about an axis of rotation, typically the elastic axis of the system. $\Lambda$ ttachment of the RSM PAPA and wing model to the tunnel sidewall turntable allows for variation of the angle of attack. Instrumentation for the $\mathrm{P} \Lambda \mathrm{P} \Lambda$ included bending and torsion strain gauges, angle-of-attack accelerometers, and three accelerometers installed on the moving plate. Model motion was measured using two linear variable differential transducers (LVDT) mounted to each side of the PAPA in order to capture plunge and pitch displacement of the system. $\Lambda$ dditional design details of the RSM PAPA are available in reference 12 .

\section{Oscillating Turntable}

The RSM was the first model to be tested using the TDT's new Oscillating Turntable (OTT). $\Lambda$ photograph of the OTT is shown in figure 3 . The OTT is essentially a very large hydraulic actuator that can be used to oscillate side-wall mounted models at arbitrary angles of attack. The TDT OTT is unique because of its ability to oscillate high inertia models (up to $65,000 \mathrm{lbm}-\mathrm{in}^{2}$ ) \pm 1 degree at frequencies up to $40 \mathrm{~Hz}$. at transonic conditions. Using the OTT, steady angles of attack and unsteady pitch oscillations can be obtained. Typically, models are oscillated at a prescribed frequency and amplitude about a mean angle of attack. The frequency response of the system is dependent on model inertia and aerodynamic loads. For the RSM, frequencies in excess of $10 \mathrm{~Hz}$. were demonstrated. Reference 13 contains details of the OTT design and operation. 
Table 1 Summary of RSM wind-tunnel tests ( $C=$ Closed, $O=O p e n)$.

\begin{tabular}{|c|c|c|c|c|c|c|c|c|}
\hline $\begin{array}{l}\text { Test } \\
\text { No. }\end{array}$ & Year & $\begin{array}{c}\text { Test } \\
\text { Medium }\end{array}$ & Mount & $\begin{array}{c}\text { Fuselage } \\
\text { Length }\end{array}$ & $\begin{array}{c}\text { Sidewall } \\
\text { Slots }\end{array}$ & $\begin{array}{c}\text { Wing/Fus } \\
\text { Gap }\end{array}$ & Nacelles & $\overline{\text { Data }}$ \\
\hline 499 & 1994 & $\mathrm{R}-12$ & $\mathrm{~B} \Lambda \mathrm{L}$ & $18 \mathrm{ft}$. & $\mathrm{O}$ & $\mathrm{O}$ & On/Off & $\Lambda \mathrm{O} \Lambda /$ Flap Polars \\
\hline 508 & 1995 & $\mathrm{R}-12$ & $\mathrm{~B} \Lambda \mathrm{L}$ & 11 & $\mathrm{O} / \mathrm{C}$ & $\mathrm{O} / \mathrm{C}$ (Foam) & On/Off & $\Lambda \mathrm{O} \Lambda /$ Flap Polars \\
\hline & & & $\mathrm{P} \Lambda \mathrm{P} \Lambda$ & 11 & $\mathrm{C}$ & C (Foam) & On/Off & Shaker Excitation \\
\hline 513 & 1995 & $\mathrm{R}-12$ & $\mathrm{~B} \Lambda \mathrm{L}$ & 18 & $\mathrm{O} / \mathrm{C}$ & C (Foam) & $\mathrm{On} / \mathrm{Off}$ & None \\
\hline 520 & 1996 & $\mathrm{R}-12$ & $\mathrm{~B} \Lambda \mathrm{L}$ & 18 & $\mathrm{O} / \mathrm{C}$ & C (Foam) & On/Off & $\Lambda \mathrm{O} \Lambda /$ Flap Polars \\
\hline 530 & 1998 & R-134a & $P \Lambda P \Lambda$ & 14 & $\mathrm{C}$ & C (Tape) & On/Off & Flutter Boundary \\
\hline & & $\mathbf{R}-134 \mathrm{a}$ & Strut & 14 & $\mathrm{C}$ & $\mathrm{C}$ (Tape) & Off & $\Lambda O \Lambda$ Polars, Tufts \\
\hline 547 & 2000 & R-134a & OTT & 14 & $\mathrm{C}$ & $\mathrm{C}$ (Tape) & Off & Wing Oscillation \\
\hline
\end{tabular}

\section{RSM Wind-Tunnel Tests}

Table 1 summarizes key aspects of the six RSM wind-tunnel tests. With the exception of TDT Test 547 , all tests were performed as part of $\mathrm{N} \Lambda \mathrm{S} \Lambda$ 's High Speed Research (HSR) program. Personnel from The Boeing Company (one of N $\Lambda \mathrm{S} \Lambda$ 's partners in the HSR program) participated in the planning and execution of these tests.

The first RSM test (499) was plagued by numerous problems with the model subsystems, but ultimately, the problems were solved and a preliminary aerodynamic database was acquired. Comparison of this data with analysis and data obtained in other wind tunnels indicated some discrepancies. The most notable difference was in the lift-curve slopes. Further study indicated that the likely cause of this discrepancy was the proximity of open slots in the TDT test section wall to the RSM wing. These open slots altered the flow over the wing by allowing flow through the wall from the high to the low pressure side.

The original objective of Test 508 was to obtain flutter data requiring the use of the shorter, lower inertia fuselage (11 foot). When it was found that this configuration would not flutter, the test objectives were changed. Test 508 explored the effects of the side-wall slots being open or closed. Additionally, the effects of leaving open and closing gaps between fuselage and wing and between fuselage and test section wall were explored. The fuselage to wing gap could be sealed with foam or tape depending whether the wing loads were being measured. $\Lambda$ small gap still existed with the use of foam. CFD results, data obtained in other wind-tunnels, in addition to data from this and the preceding RSM wind-tunnel test indicated that the most appropriate configuration was slots closed and gaps sealed.

Test 513 was supposed to use the lessons learned from the preceding tests to acquired a large, high quality, aerodynamic database. Unfortunately, the RSM delaminated before any significant data was acquired. $\Lambda$ fter the model was repaired, Test 520 successfully acquired this aerodynamic data.

The last two tests used a new; aerodynamically im- proved fuselage. Additionally, the new fuselage had a different center of rotation and center of gravity so that RSM/P AP $\Lambda$ flutter data could be acquired. Test 530 acquired flutter data with and without engine nacelles. The most recent test of the RSM was Test 547 where the model was oscillated on the TDT's Oscillating Turntable.

While significant lessons were learned on the first three tests of the RSM, only the last three tests are considered to have been successful in generating quality aerodynamic data for code validation. Data from these tests are available for dissemination.

\section{RSM Experimental Data}

The RSM data acquired during Test 520 (balance) has been thoroughly documented in reference 14 . Reference 12 summarizes several HSR tests including RSM Test 530 (PAP 4 ), but it did not examine any of the unsteady aerodynamic data. $\Lambda$ dditionally, both the balance and P $\Lambda P \Lambda$ mounts have a limited angleof-attack range due to load and deflection limits. The larger load limits on the OTT allowed mean angles of attack up to 12 degrees. The focus of this section of the paper will be to document the aerodynamic data acquired during Test 547, including steady pressures and a comparison with some data from Test 520 (balance). Unsteady pressures from Test 547 will also be examined and where appropriate compared with ap-

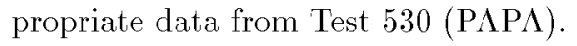

\section{OTT Database Description}

The RSM/OTT database is comprised primarily of data acquired at various combinations of two dynamic pressures $(q)$ and ten Mach numbers $(M)$. The dynamic pressures were 100 and 150 psf, and the Mach numbers were $0.5,0.7,0.8,0.9,0.95,0.98,1.0,1.02$, 1.05, and 1.1. Table 2 summarizes the various combinations of mean angle of attack $\left(\alpha_{0}\right)$, oscillation frequency $(f)$, and amplitude $\left(\alpha_{1}\right)$ at which data were acquired. Note that at each combination of $M, q$, and $\alpha_{o}$, a steady data point (i.e. $f=0 \mathrm{~Hz}$.) was acquired. The length of each time history was either 15 or 30 seconds depending on the frequency of oscillation. $\Lambda \mathrm{d}-$ ditionally, at each combination of $M$ and $q$, data were 
acquired during a sine sweep from 1 to $12 \mathrm{~Hz}$. Other data acquired during the RSM/OTT wind-tunnel test included $M, q$, and $\alpha_{o}$ conditions corresponding to the flutter points obtained from Test 530 (RSM/P AP $\Lambda$ ).

Table 2 Pitch oscillation amplitudes ( $\alpha_{1}, \pm$ Deg.) acquired for various mean angles of attack $\left(\alpha_{o}\right.$, Deg.) and frequency $(f, \mathrm{~Hz}$.) combinations for Test 547 (OTT).

\begin{tabular}{ccccccc}
\hline \hline$\alpha_{o}$ & $f=0$ & $f=1$ & $f=2$ & $f=5$ & $f=8$ & $f=10$ \\
\hline-5 & 0 & 2 & 1 & 1 & 0.5 & $0.25,0.5$ \\
-3 & 0 & 2 & 1 & 1 & 0.5 & $0.25,0.5$ \\
0 & 0 & 2 & 1 & 1 & 0.5 & $0.25,0.5$ \\
0 & 0 & 3 & 1.5 & 1.5 & 1 & $0.5,1.0$ \\
3 & 0 & 2 & 1 & 1 & 0.5 & $0.25,0.5$ \\
6 & 0 & 2 & 1 & 1 & 0.5 & $0.25,0.5$ \\
9 & 0 & 2 & 1 & 1 & 0.5 & $0.25,0.5$ \\
12 & 0 & 2 & 1 & 1 & 0.5 & $0.25,0.5$ \\
15 & 0 & 2 & 1 & 1 & 0.5 & $0.25,0.5$ \\
\hline \hline
\end{tabular}

\section{Steady Data}

Figures 4 and 5 show examples of mean pressure coefficients acquired on the OTT at several fixed angles of attack $(f=0 \mathrm{~Hz}$.). The data shown in figure 4 is for a subsonic Mach number of 0.5 and all the pressure coefficients are well below $C_{p}$ critical $\left(C_{p_{c r}}=-2.38\right)$. Thus, none of the features in the data can be attributed to shock waves. $\Lambda$ ll span stations exhibit a variation of the leading edge suction peak with angle of attack. The most interesting features of this data can be found at 30 and $60 \%$ span. Here the effects of vortical flow can bee seen at the larger magnitude angles of attack. At $60 \%$ span, vortical flow is noted on the lower surface from the leading edge to $20 \%$ chord for -5 and -3 degrees, and on the upper surface for angles of 6 , 9 , and 12 degrees. $\Lambda$ this Mach number the zero lift angle of attack for the RSM is approximately 1.6 degrees, and the pressures at this span station display reasonable symmetry with respect to that angle. $\Lambda \mathrm{t}$ $30 \%$ span, vortical flow is noted for 12 degrees and to a lesser extent and further aft, vortical flow is shown in the -5 degree data. The $10 \%$ span and $95 \%$ span stations exhibit no significant features with the exception of the -5 degree data at $10 \%$ span. Here, vortical flow appears to be present on the lower surface at $10 \%$ chord. The potential significance of this relative to RSM/P $\Lambda P \Lambda$ flutter will be discussed later.

The data shown in figure $\overline{5}$ was acquired at a Mach number of 0.95 . In general, the major features of this data are very similar to the Mach 0.5 data. The primary difference is that the pressure distributions tend to be flatter. This observation is consistent with the general tendency for the pressure distribution to flatten out with increased Mach number for this type of planform. For this type of configuration, shocks generally occur near the trailing edge of the wing. Although

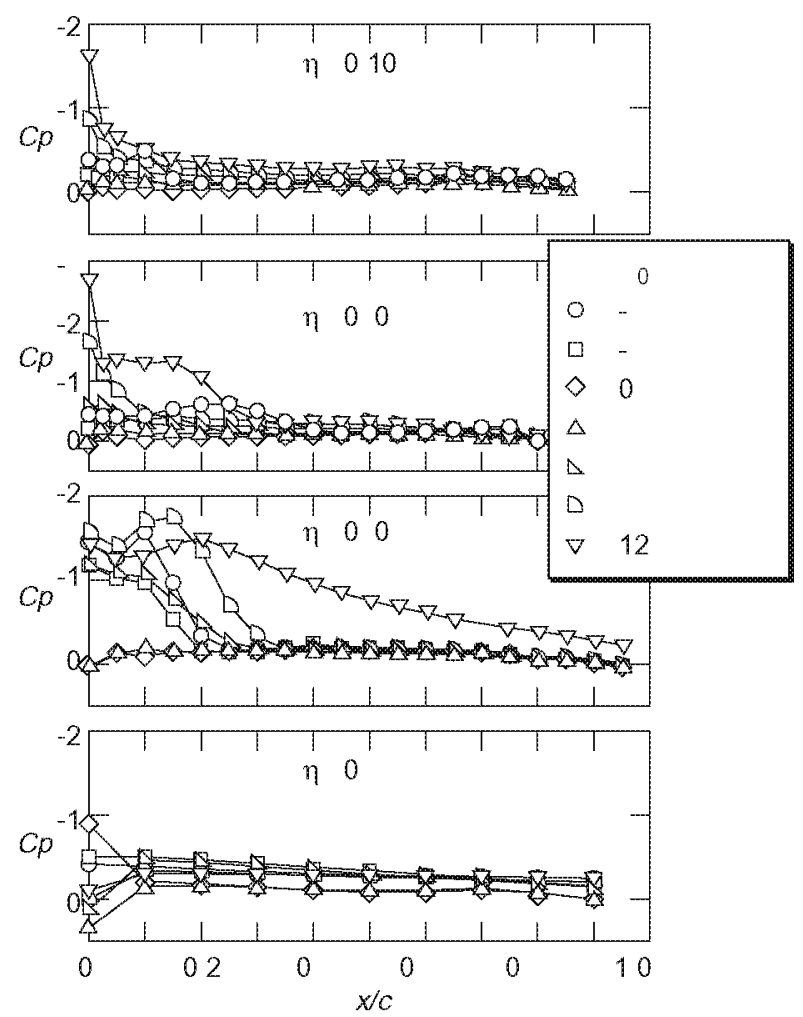

Fig. 4 Comparison of Test 547 (OTT) steady pressure coefficients (suction side only) for seven angles of attack. $M=0.50$ and $q=150$ psf.

difficult to see in this plot, shocks are present at the $60 \%$ span station and $85 \%$ chord for some angles of attack.

To evaluate data consistency between wind-tunnel tests, two conditions were selected for comparison of steady data between Test 547 (OTT) and Test 520 (balance). The data points selected were for the highest angle-of-attack conditions where consistent tunnel conditions ( $M$ and $q$ ) could be found. Figure 6 is for Mach 0.7 and an angle of attack of 6 degrees, and figure 7 is for Mach 0.95 and an angle of attack of 3 degrees. Overall, these data sets compare very well in spite of several known differences between the two wind-tunnel tests. One difference was that Test 547 used R-134a and Test 520 used R-12 as the test medium. The change in gas resulted in a change in the Reynolds number of about $2 \%$ in the subsonic case and about $7 \%$ in the transonic case. The other difference was that the 18-foot fuselage was used in Test 520 and the 14-foot fuselage was used in Test 547. This fuselage difference is the likely cause of some of the differences between the two data sets, especially for the $10 \%$ span data.

\section{Unsteady Data}

Figures 8 and 9 show samples of unsteady pressure data acquired during Test 547 (OTT). Magnitude and phase of $C_{p}$ at a forced frequency of $2 \mathrm{~Hz}$. and an os- 

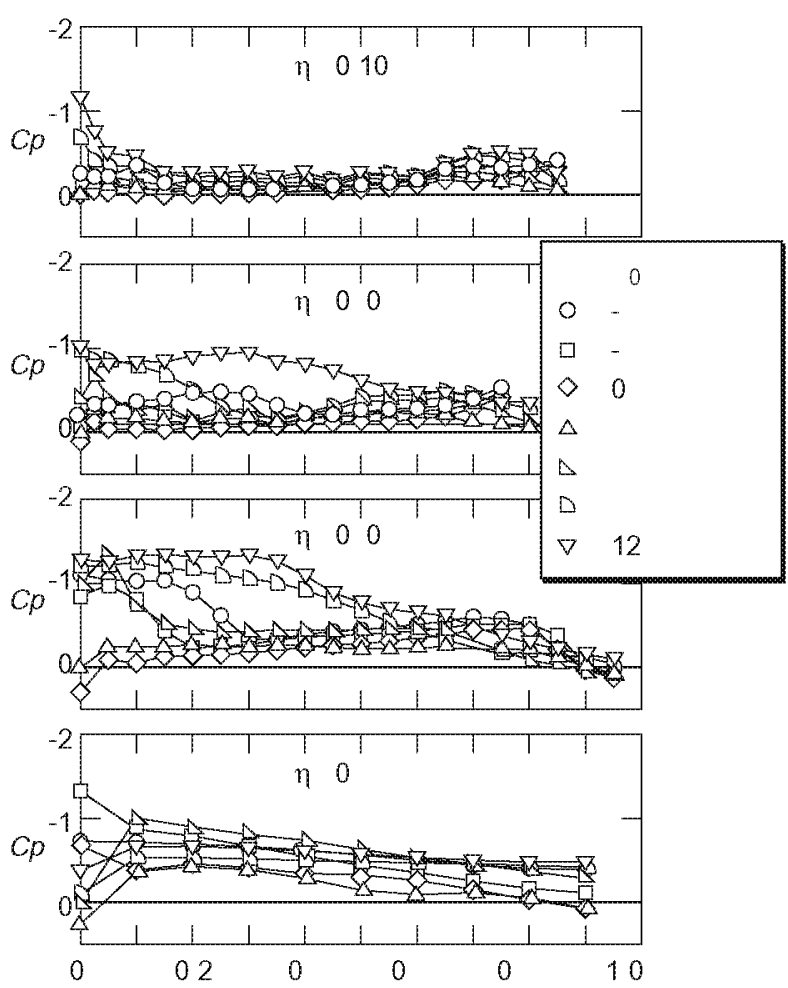

Fig. 5 Comparison of Test 547 (OTT) steady pressure coefficients (suction side only) for seven angles of attack. $M=0.95$ and $q=150$ psf.

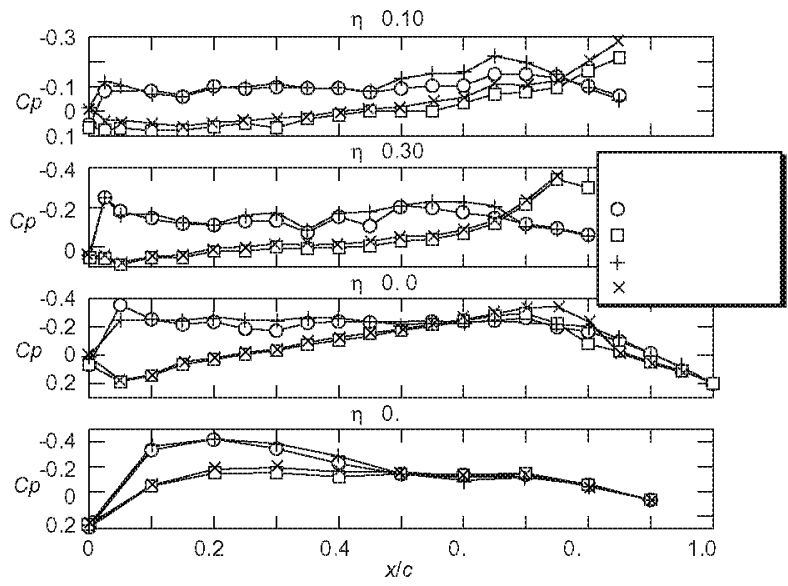

Fig. 6 Comparison of Test 520 (BAL) and Test 547 (OTT) mean pressure coefficient. $M=0.70$, $q=150$ psf, $\alpha_{o}=6$ deg., $R c_{B A L}=\mathbf{3 . 1 9} \times 10^{6} / \mathrm{ft}$, and $R \operatorname{RoTT}_{T}=\mathbf{3 . 1 3} \times \mathbf{1 0 ^ { 6 }} / \mathbf{f t}$.

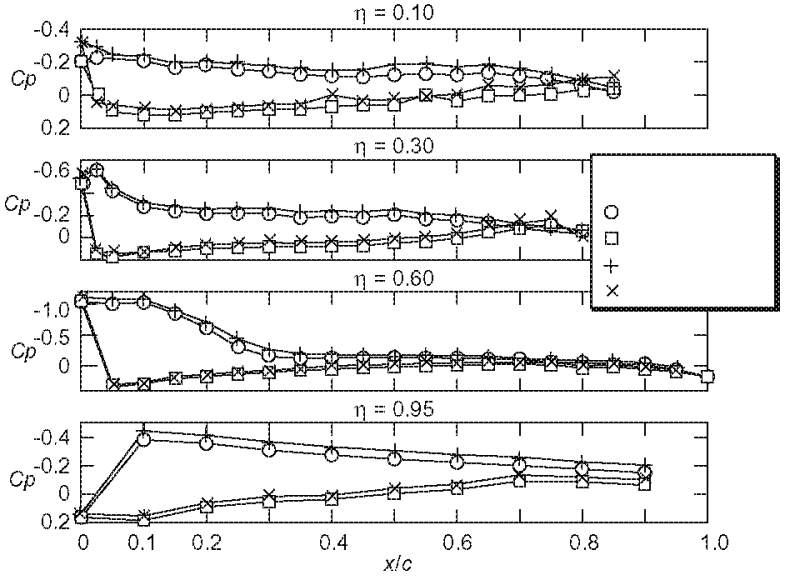

Fig. 7 Comparison of Test 520 (BAL) and Test 547 (OTT) mean pressure coefficient. $M=\mathbf{0 . 9 5}$, $q=150$ psf, $\alpha_{o}=\mathbf{3}$ deg., $R_{c_{B A L}}=\mathbf{2 . 3 6} \times \mathbf{1 0} 0^{6} / \mathrm{ft}$ and $R_{\text {OTT }}=\mathbf{2 . 2 1} \times \mathbf{1 0} 6 \mathrm{ft}$.

cillation amplitude of \pm 1 degree are shown for three angles of attack at a subsonic and a transonic Mach number. High magnitudes in this type of data are generally associated with shock motion or movement of vortical flow regions. In both sets of data, large magnitudes are noted at the leading edge for the nonzero angles of attack. In the subsonic data, the effects of vortex motion are seen primarily at 30 and $60 \%$ span. There may be a small amount of vortex motion near the leading edge at $95 \%$ span for 6 degrees, and no vortex motion is noted at $10 \%$ span. The transonic data in figure 9 shows many of the same features that were found in the subsonic data. In general, the major features found in the subsonic data are moved further aft in the transonic data. Additionally, several new features are noted near the leading edge for 10 and $30 \%$ span. Examination of $C_{p}$ critical values $\left(C_{p_{c r}}=-\right.$ 0.100 ) indicates that these features are probably not associated with shock waves but are probably the result of vortical flow. The magnitude peak hinted at in $95 \%$ span, subsonic data is more pervasive here.

Some of the data from Test 530 (PAPA) will now be examined and compared with corresponding data from Test 547 (OTT). The flutter points and boundaries for the RSM/P $\mathrm{P} \Lambda$ configuration are shown in figure 10. These results were for a clean wing, but similar results were also found with the engine nacelles installed. Time history data were acquired at each of the flutter points. The results are plotted as dynamic pressure versus Mach number for various values of the mean angles of attack. The baseline ( 0 degrees) flutter boundary exhibits a shallow transonic dip followed by an abrupt rise. $\Lambda$ s angle of attack becomes more negative, the boundaries shift to lower dynamic pressures and tend to flatten out. The flutter frequency for these results varied from 4.75 to $4.78 \mathrm{~Hz}$. The results presented in figure 10 were unexpected for what 

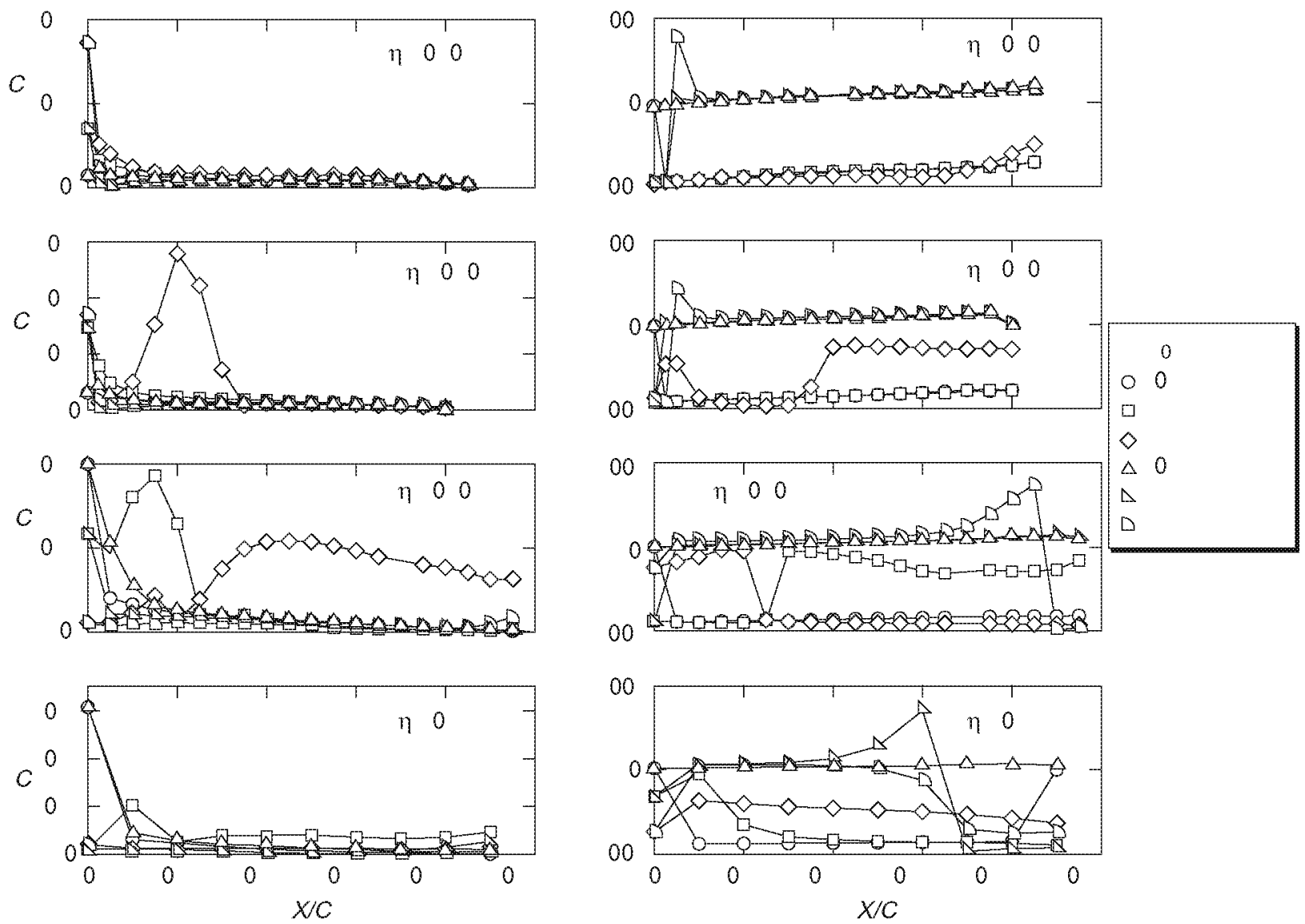

Fig. 8 Comparison of Test 547 (OTT) unsteady pressures at three mean angles of attack. $M=0.50, q=150$ psf., $f=\mathbf{2} \mathrm{Hz}$., and $\alpha_{1}= \pm 1 \mathrm{deg}$.

was considered a simple 2 degree-of-freedom configuration. The flutter results indicate a strong dependence on angle of attack which is unusual for thin wings at subsonic conditions.

The strong relationship between the flutter boundary and angle of attack may be due in large part to the airfoil shape on the strake portion of the wing. While the outboard wing has a sharp leading edge, the strake portion of the wing has a rounded leading edge but has a relatively flat lower surface. Because of this strake geometry negative angles of attack would have a greater tendency to generate vortical flow further forward and inboard on the lower surface of the strake than positive angles on the upper surface. This hypothesis is supported by the steady data shown in figure 6 where there is evidence of a lower surface strake vortex at $10 \%$ span for -5 degrees angle of attack. For this span station there is no evidence of vortical flow for any of the positive angles up to 12 degrees. Similarly for the $30 \%$ span there is evidence of a lower surface strake vortex at -5 and, to a lesser extent, -3 degrees. $\Lambda$ this span station, evidence of an upper surface vortex exists only at 12 degrees. The formation of the strake vortex on the lower surface

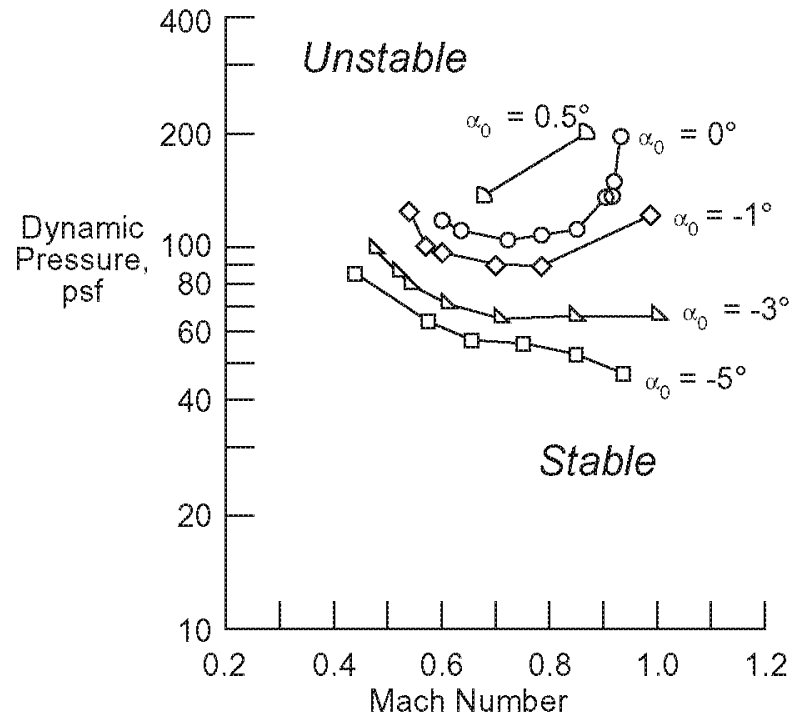

Fig. 10 Experimental flutter boundaries as a function of mean angle of attack for the RSM/PAPA configuration, no nacelles. 

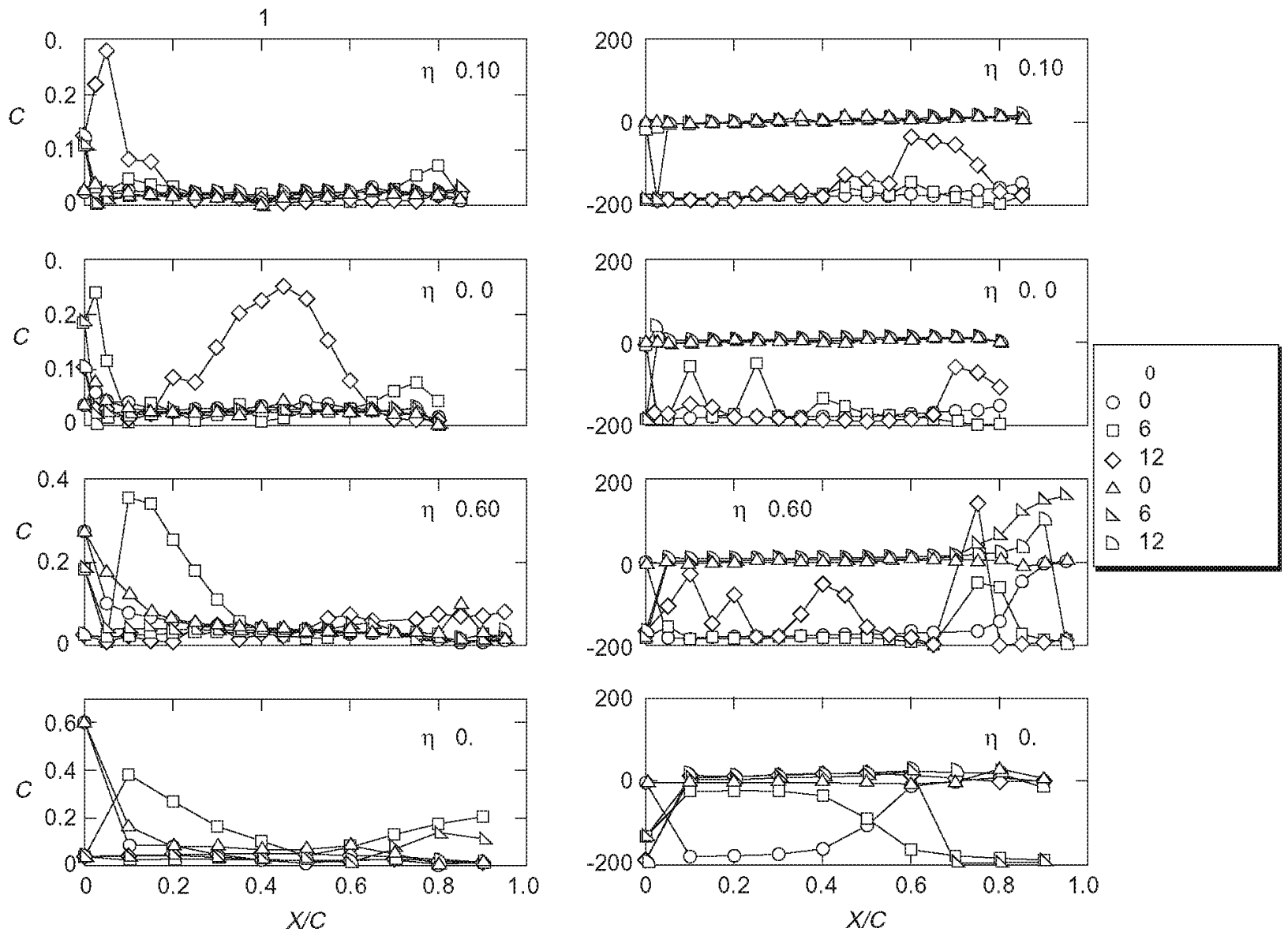

Fig. 9 Comparison of Test 547 (OTT) unsteady pressures at three mean angles of attack. $M=0.95, q=150$ psf., $f=\mathbf{2} \mathrm{Hz}$., and $\alpha_{1}= \pm 1 \mathrm{deg}$.

as angle of attack is reduced to - $\overline{0}$ degrees would cause the center of pressure to move forward and apparently, had a destabilizing effect on the flutter boundary.

Figure 11 shows a comparison between unsteady pressure data acquired during Test 530 (P $\Lambda \mathrm{P} \Lambda)$ and data acquired at approximately the same flow conditions during Test 547 (OTT). The P $\Lambda \mathrm{P} \Lambda$ data shown is for the Mach $0.93,-5$ degrees angle of attack, flutter point shown in figure 10 . Here, the OTT data approximately replicates the flow features found in the flutter data. Considering the known differences between the two test conditions, the agreement is very reasonable. While the flutter mode observed on PAPA was dominated by pitching motion, the flutter mode did include a plunging component and the center of rotation was not at the $\mathrm{P} \Lambda \mathrm{P} \Lambda$ shear center. In contrast, the OTT data was for pure pitching oscillation about a fixed center of rotation. Another difference was that the amplitude of oscillation for the $\mathrm{P} \Lambda \mathrm{P} \Lambda$ data was increasing during data acquisition while the OTT data was acquired for a fixed amplitude oscillation. Finally, there was an angle-of-attack difference due to aeroelastic twist of $\mathrm{P} \Lambda \mathrm{P} \Lambda$. The reported angle of attack for

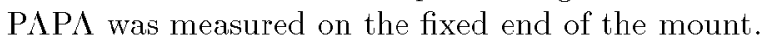

Examination of all the $\mathrm{P} \Lambda \mathrm{P} \Lambda$ flutter data and corresponding OTT data indicate that the best correlation between the two data sets is for -5 degrees angle of attack. For the -3 degree angle-of-attack data the 30 and $60 \%$ span data generally agree while the 10 and $95 \%$ data show poor correlation. The correlation for the -1 , 0 and 0.5 degree data is generally poor. One possible explanation for these discrepancies is the difference in angle of attack due to $\mathrm{P} \Lambda \mathrm{P} \Lambda$ twist. The key features of the flow are probably not as sensitive to small differences in angle of attack when the magnitude of the angle is large.

\section{CFD Analysis}

There is significant interest in using the RSM data, acquired during its various tests, for validating computational methods. $\Lambda$ primary reason for this interest is the unusual flutter boundary (figure 10) acquired during testing of the RSM on the P $\Lambda \mathrm{P} \Lambda$. As previously mentioned, high-aspect ratio wings with thin airfoils exhibit no variation in the flutter dynamic pressure due to moderate changes in angle of attack at subsonic conditions. This characteristic allows the use of linear flutter analysis methods based on lifting surface 

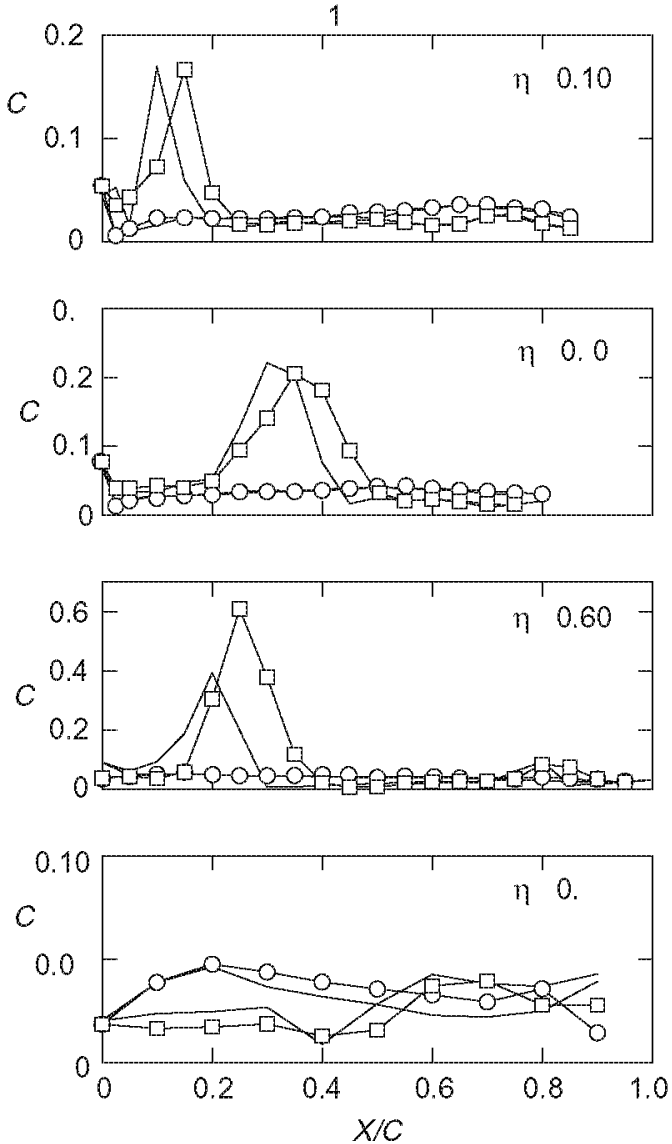
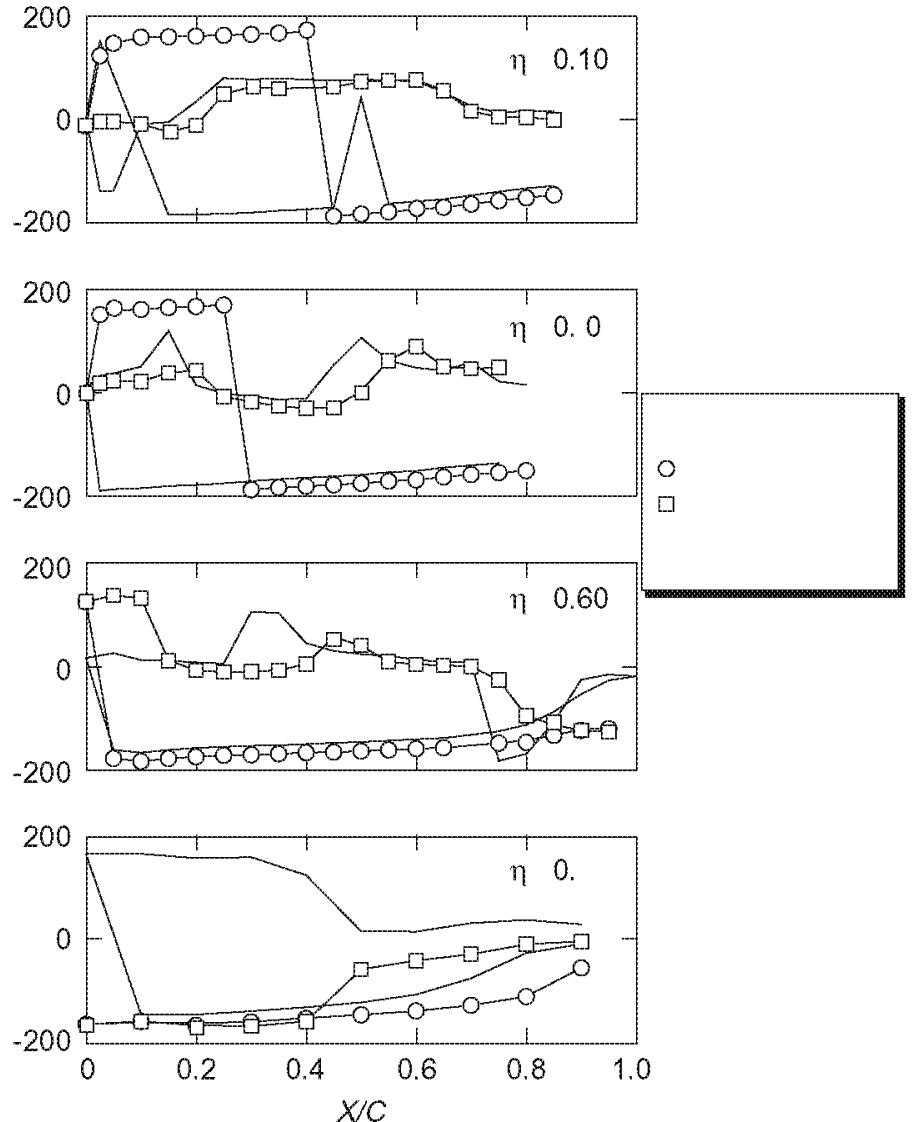

Fig. 11 Comparison of OTT and PAPA unsteady pressure data. $M=\mathbf{0 . 9 3 4 ,} q=48$ psf, $\alpha_{O_{O T T}}=-5$ deg., $\alpha_{O_{P A P A}}=-4.75 \mathrm{deg} ., f_{O T T}=4.87 \mathrm{~Hz} ., \alpha_{1}=0.5 \mathrm{deg} .$, and $f_{P A P A}=4.74 \mathrm{~Hz}$.

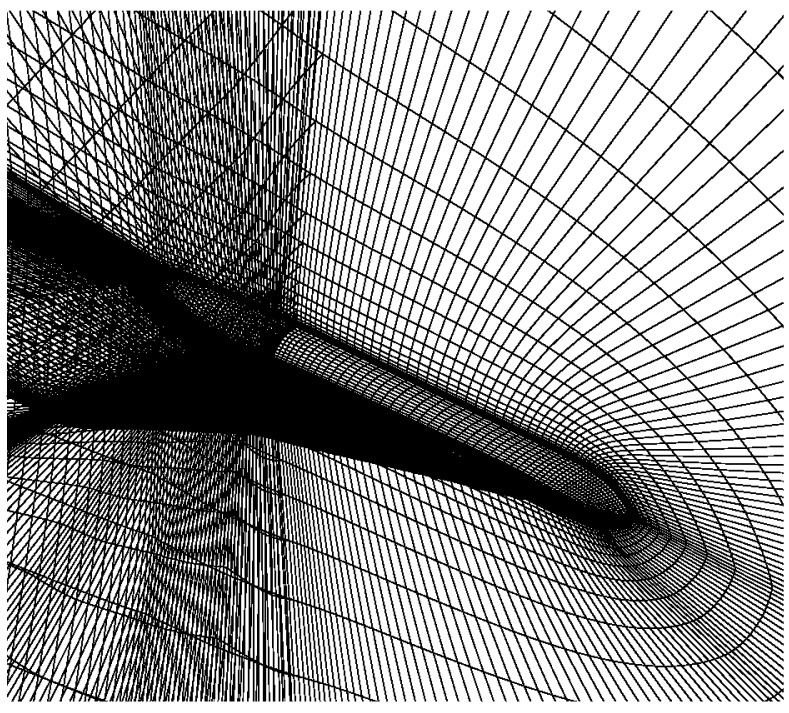

Fig. 12 Computational grid for RSM with 14-foot fuselage. theories (flat plate models). But the flutter boundary exhibited by the RSM on the PAPA is indicative of significant nonlinear effects since the flutter boundary is a strong function of angle of attack across the Mach number range. Therefore, since there are no shocks at subsonic conditions, the nonlinear effects must be due to vortical flow induced by the RSM's low aspect ratio, high inboard sweep, and outboard sharp leading edge. $\Lambda$ s a result of this aeroelastic sensitivity to complex flow physics, this dataset poses an interesting challenge to the validation of computational methods.

\section{CFL3Dv6 and Grid}

The recently-developed CFL3D version 6.0 (CLF3Dv6) computational fluid dynamics (CFD) code is being used for the steady and unsteady analysis of the RSM wind-tunnel model. The CFL3Dv6 code solves the time-dependent conservation law form of the Reynolds-averaged Navier-Stokes equations ${ }^{15}$ using a finite-volume approach. Upwind-biasing is used for the convective and pressure terms while central differencing is used for the shear stress and heat transfer terms. Implicit time advancement is used with the ability to solve steady or unsteady flows. Subiteration and multigrid capabilities are 

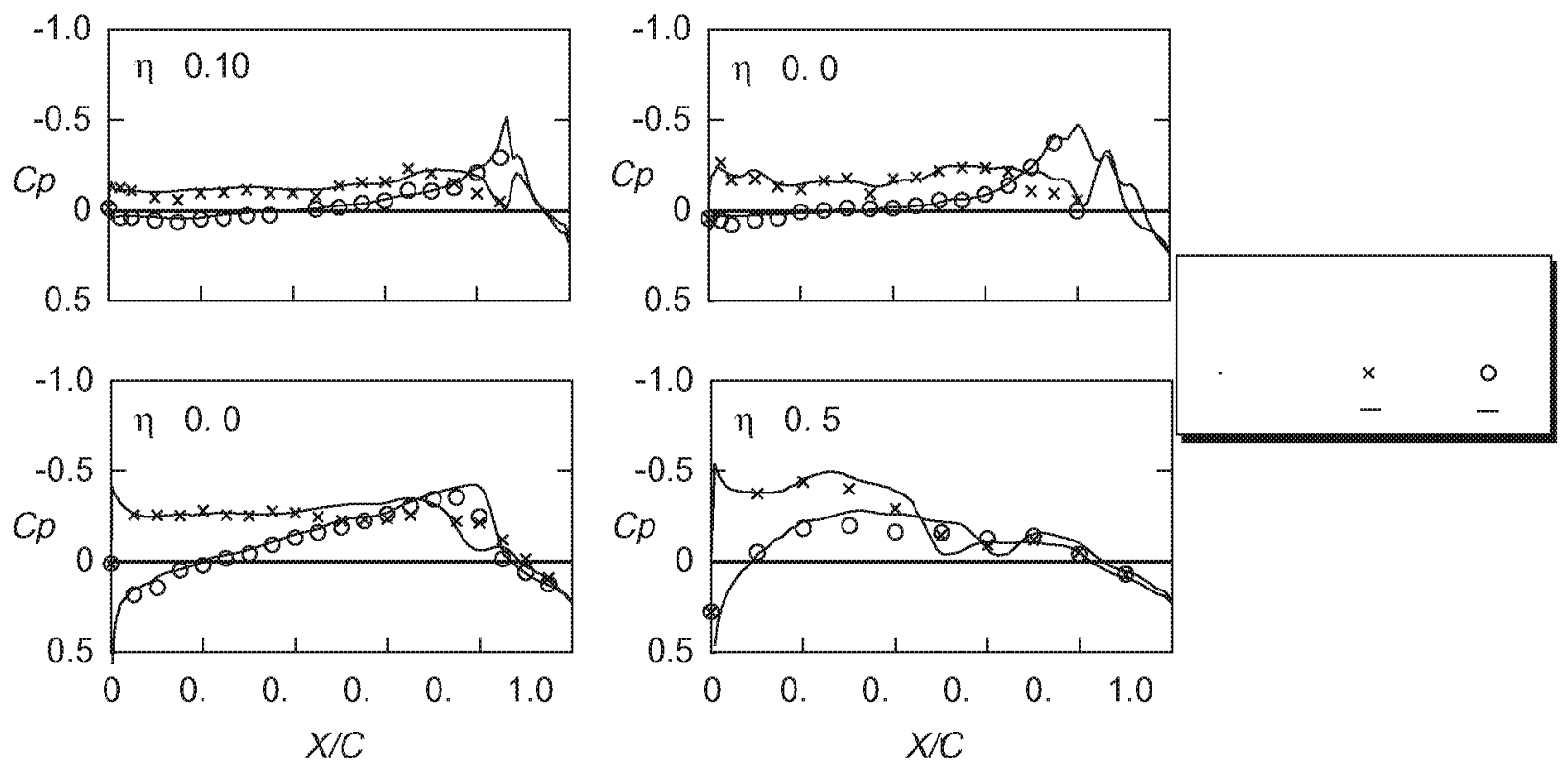

Fig. 13 Comparison of CFL3D analysis and Test 547 (RSM/OTT) steady pressure coefficients. $M=0.95$, $q=150$ psf, $R_{c}=2.2 \times 10^{6} / \mathrm{ft}$, and $\alpha_{o}=3 \mathrm{deg}$.
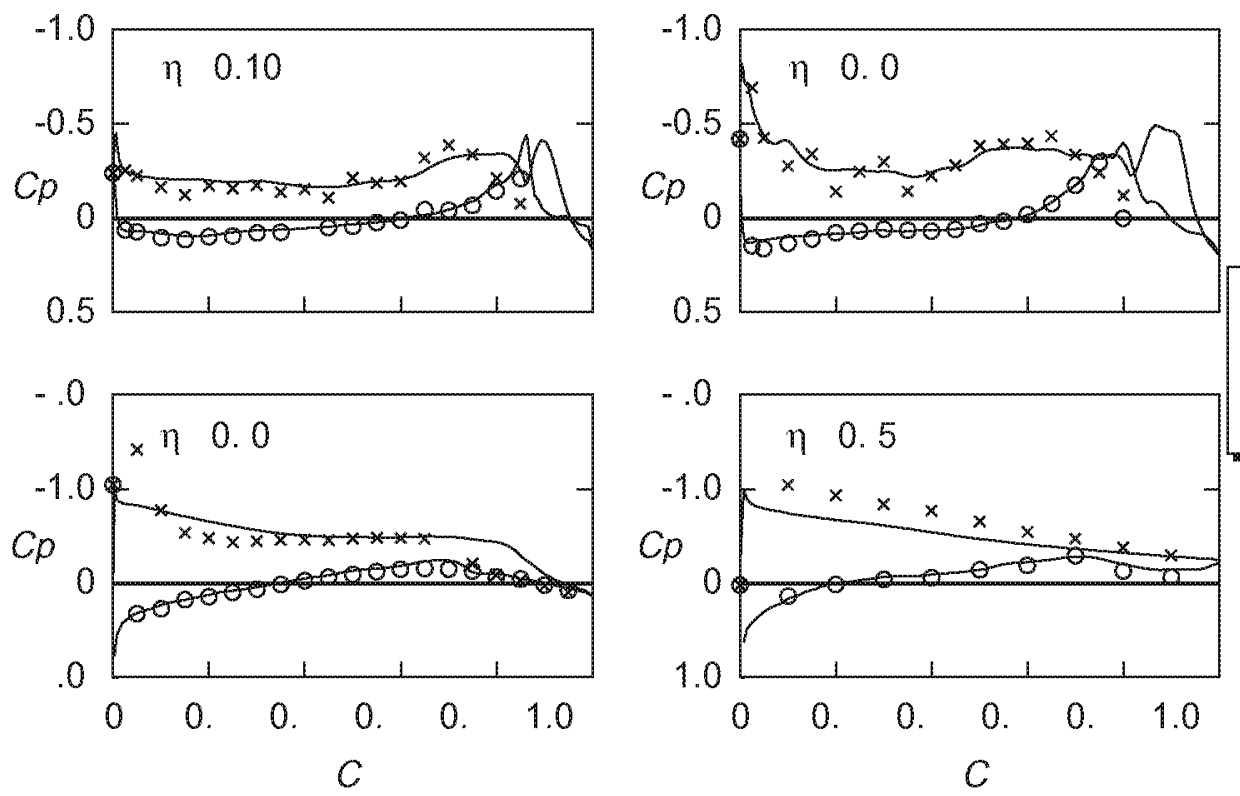

Fig. 14 Comparison of CFL3D analysis and Test 547 (RSM/OTT) steady pressure coefficients. $M=0.95$, $q=150 \mathrm{psf}, R_{c}=2.2 \times 10^{6} / \mathrm{ft}$, and $\alpha_{o}=6 \mathrm{deg}$. 

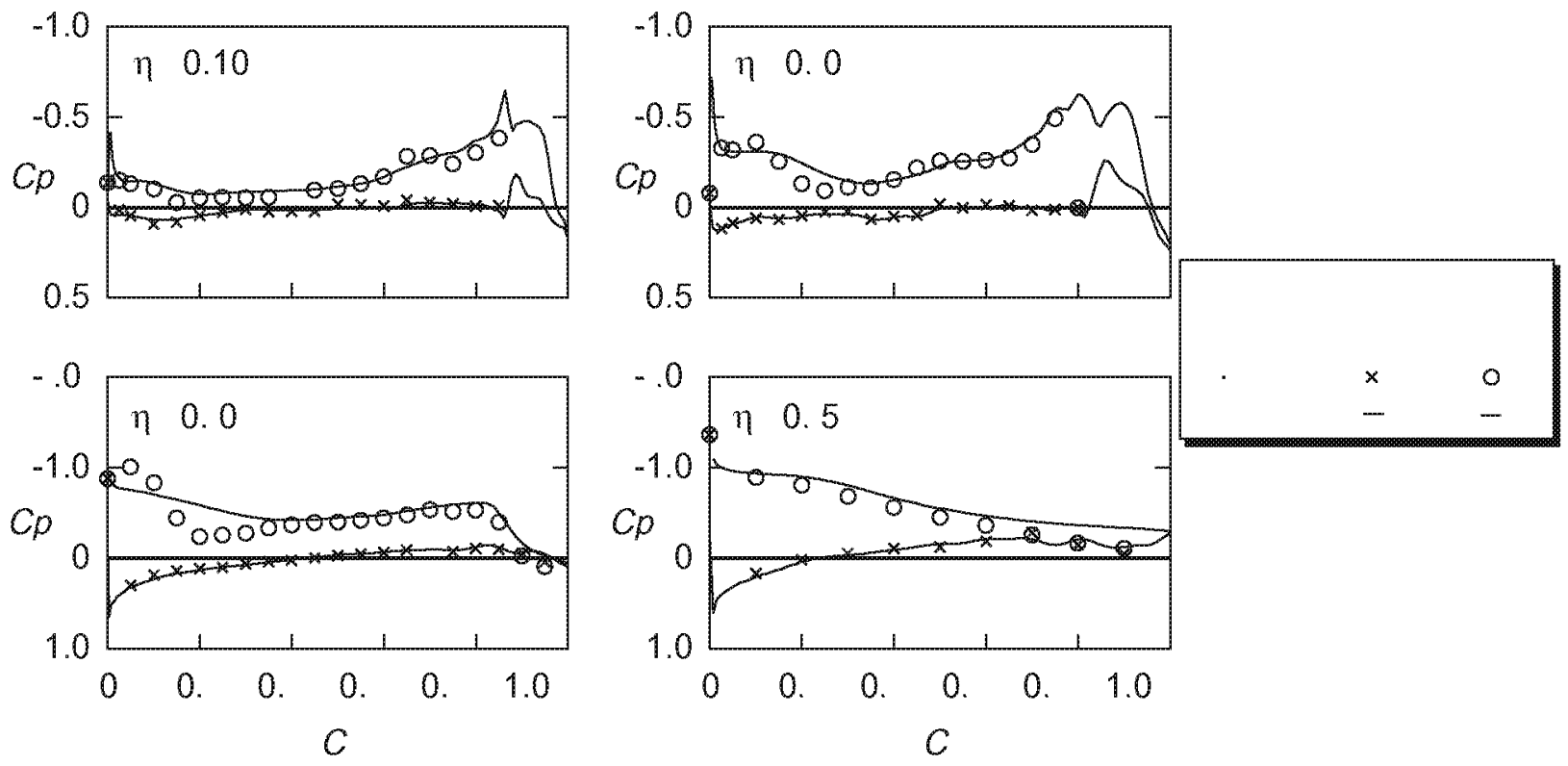

Fig. 15 Comparison of CFL3D analysis and Test 547 (RSM/OTT) steady pressure coefficients. $M=0.95$, $q=150 \mathrm{psf}, R_{c}=2.2 \times 10^{6} / \mathrm{ft}$, and $\alpha_{o}=-3 \mathrm{deg}$.
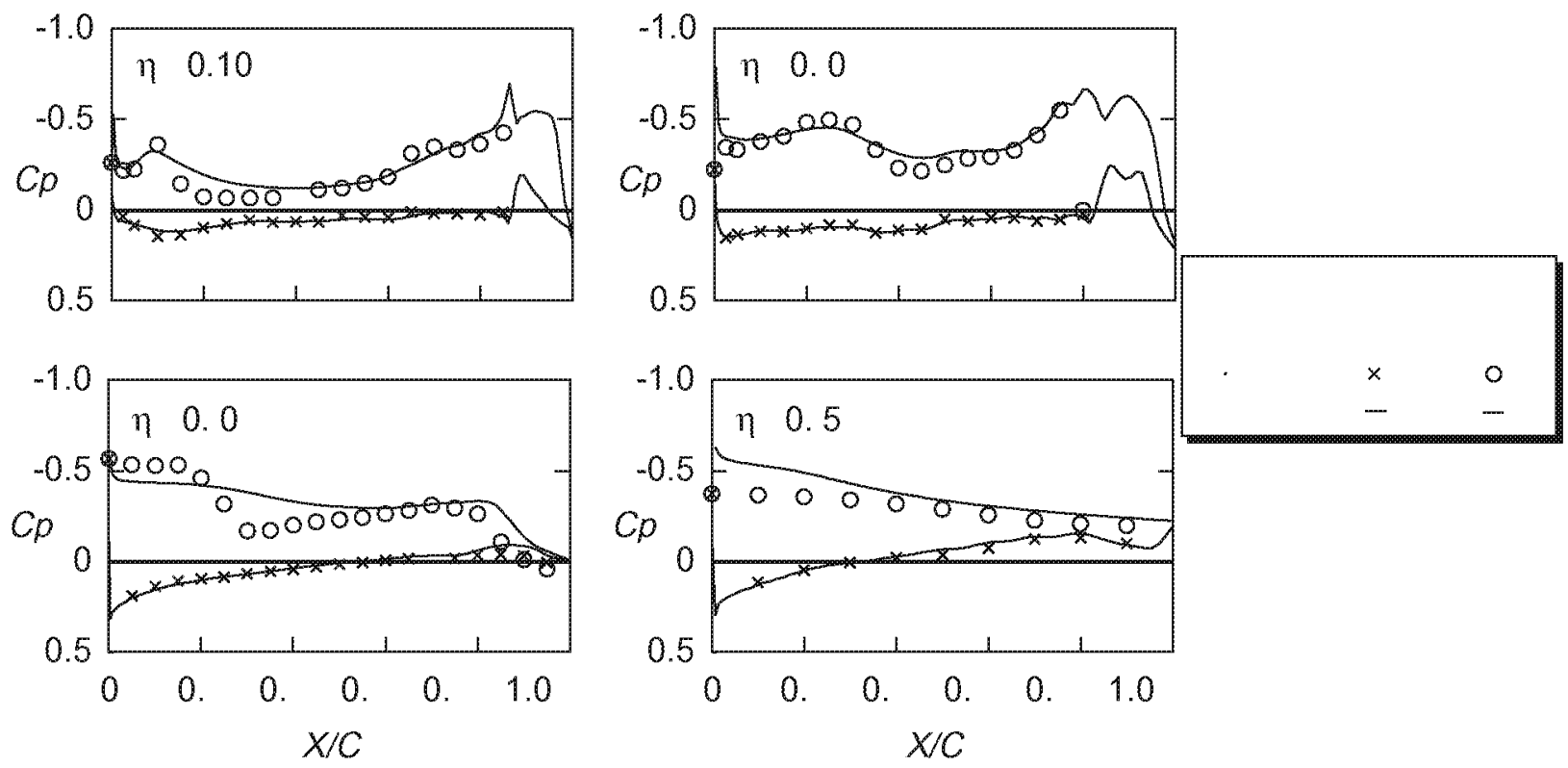

Fig. 16 Comparison of CFL3D analysis and Test 547 (RSM/OTT) steady pressure coefficients. $M=0.95$, $q=150 \mathrm{psf}, R_{c}=2.2 \times 10^{6} / \mathrm{ft}$, and $\alpha_{o}=-5 \mathrm{deg}$. 
available for improved accuracy and convergence acceleration. In addition, numerous turbulence models are provided. ${ }^{16}$

Data from photogrammetry, used to measure surface ordinates, was used to generate IGES models of the RSM and the 14-foot fuselage. These IGES models were then used to create grids for subsequent use in CFD analyses. One such grid is shown in figure 12. It is a $\mathrm{C}-\mathrm{H}$ topology grid dimensioned $305 \times 81 \times 49$ grid points, suitable for Navier-Stokes calculations.

\section{Computational Results and Comparisons with Experimental Data}

$\Lambda$ comparison of computational and experimental steady pressure distributions at a Mach number of 0.95 , a $R e=2.2 \times 10^{6} / \mathrm{ft}$, and $\alpha_{o}=3,6,-3$, and -5 degrees will be shown in this section. $\Lambda$ ll results were computed using the Spalart- $\Lambda$ llmaras turbulence model.

Figure 13 presents the steady pressure distributions at 3 degrees angle of attack. The results compare favorably at all span stations. The large variations in the computational results at the 10 and $30 \%$ span stations near the trailing edge are due to surface variations associated with the trailing edge control surface. The control surface was not instrumented with pressure ports so there are no pressure measurements available for this region. $\Lambda$ t the $60 \%$ span station, the shock was predicted to be farther aft than the test measurement indicates. At $95 \%$ span, the general variations in the flow physics appear to have been adequately captured.

The comparison of steady pressure distributions at 6 degrees angle of attack are presented in Figure 14. $\mathrm{\Lambda t}$ this condition, larger discrepancies between the computational and experimental results are seen. Again, the inboard span stations correlate well but significant differences are seen for the two outboard stations, with the largest discrepancies occurring on the suction surface. This lack of correlation for the outboard stations is persistent at other angles of attack as well.

Figure 15 shows the results for -3 degrees angle of attack. The comparison for the two inboard stations is quite good including the presence of mild vortical flow at the $30 \%$ span station near the leading edge. The comparison at the $60 \%$ span station is poor as the computation does not capture the flow phenomena near the leading edge.

Finally, figure 16 presents the comparison for -5 degrees angle of attack. Once again, the comparison with the two inboard span stations is reasonable. In particular, the vortical flow near the quarter-chord at the $30 \%$ span station has been captured by the computations. The comparison with the outboard span stations has deteriorated, especially at the $60 \%$ station. The cause of this discrepancy is not yet known and additional analyses are needed. It may be necessary to perform analysis forcing transition at a location consistent with the location of the transition strip on the
RSM $^{17}$ Improved grid resolution may also be needed for the outboard span stations in order to capture the vortical flow phenomena in that region. ${ }^{17}$

In a previous section it was stated that the tendency of the lower surface to generate vortical flows inboard at moderate negative angles of attack might be a contributing factor to the unusual flutter boundary presented in figure 10 . This characteristic appears to be supported by the present analyses (figures 15 and 16), at least for the inboard sections. However, to fully understand the effect of the vortical flow on the flutter boundary of the RSM, the flow physics at the outboard stations needs adequate resolution. $\Lambda \mathrm{d}$ ditional analyses are currently underway to address these issues.

\section{Concluding Remarks}

$\Lambda$ large database of steady, unsteady, and flutter wind-tunnel data has been obtained for three configurations based on an HSCT design: the Rigid Semispan Model (RSM) on a balance, the RSM on a Pitch and


cillating Turntable (OTT). The database covers an extensive Mach number range from subsonic to low supersonic with a special focus on transonic conditions. The RSM was highly instrumented and the acquired database represents one of the largest aerodynamic and aeroelastic databases available. Examples of steady and unsteady pressure data were shown and compared with other RSM wind-tunnel data sets. The flutter behavior of the RSM on the P $\mathrm{P} \Lambda$ mount was examined. Preliminary CFD analyses were performed and compared with experimental data. $\Lambda$ ll of the RSM wind-tunnel data acquired is available for public distribution.

\section{Acknowledgements}

The authors would like to acknowledge the support of Mr. Norm Willey for his work acquiring the photogrammetry data and the support of the GEOL $\triangle B$ at N $\Lambda \mathrm{S} \Lambda$ Langley Research Center where the CFD grid for the RSM was generated.

\section{References}

${ }^{1}$ Ruiz-Calavera, L. P. and et al, "A New Compendium of Unsteady Aerodynamic Test Cases for CFD: Summary of AVT WG-003 Activities," International Forum on Aeroelasticity and Structural Dynamics, June 1999.

${ }^{2}$ Bennett, R. M., Eckstrom, C. V., Rivera, Jr, J. A., Dansberry, B. E., Farmer, M. G., and Durham, M. H., "The Benchmark Aeroelastic Models Program: Description and Highlights of Initial Results," NASA TM 104180, April 1991.

${ }^{3}$ Durham, M. H., Keller, D. F., Bennett, R. M., and Wieseman, C. D., "A Status Report on a Model for Benchmark Active Controls Testing," AIAA/ASME/ASCE/AHS/ASC 32nd Structures, Structural Dynamies, and Materials Conference, No. 91-1011, Baltimore, MD, April 1991.

${ }^{4}$ Rivera, Jr, J. A., Dansberry, B. E., Bennett, R. M., Durham, M. H., and Silva, W. A., "NACA0012 Benchmark Model Experimental Flutter Results with Unsteady Pressure Distributions," NASA TM 107581, April 1992. 
${ }^{5}$ Rivera, Jr, J. A., Dansberry, B. E., Durham, M. H., Bennett, R. M., and Silva, W. A., "Pressure Measurements on a Rectangular Wing with a NACA0012 Airfoil During Conventional Flutter," NASA TM 104211, July 1992.

${ }^{6}$ Dansberry, B. E., Durham, M. H., Bennett, R. M., Turnock, D. L., Silva, W. A., and Rivera Jr, J. A., "Physical Properties of the Benchmark Models Program Supercritical Wing," NASA TM 4457, Sept. 1993.

${ }^{7}$ Scott, R. C., Hoadley, S. T., Wieseman, C. D., and Durham, M. H., "The Benchmark Active Controls Technology Model Aerodynamic Data," Proceedings of the 95th Aerospace Sciences Meeting and Exhibit, No. 97-0829, Reno, NV, Jan. 1997.

${ }^{8}$ Schuster, D. M., Spain, C. V., and et al, "Development, Analysis, and Testing of the High Speed Research Flexible Semispan Model," NASA CR 1999-209556, Sept. 1999.

${ }^{9}$ Corliss, J. M. and Cole, S. R., "Heavy Gas Conversion of the NASA Langley Transonic Dynamics Tunnel," Proceedings of the 20th Advanced Measurements and Ground Testing Technology Conference, No. 98-2710, Albuquerque, NM, June 1998.

${ }^{10}$ Cole, S. R. and Rivera Jr, J. A., "The New Heavy Gas Testing Capability in the NASA Langley Transonic Dynamics Tunnel," Royal Aeronautical Society Wind Tunnels and Wind Tunnel Test Techniques Forum, No. 4, Cambridge, UK, April 1997.

${ }^{11}$ Farmer, M. G., "A Two-Degree-of-Freedom Flutter Mount System with Low Damping for Testing Rigid Wings at Different Angles of Attack," NASA TM 83302, 1982.

${ }^{12}$ Silva, W. A., Keller, D. F., Florance, J. R., Cole, S. R., and Scott, R. C., "Experimental Steady and Lnsteady Aerodynamic and Flutter Results for HSCT Semispan Models," AIAA/ASME/ASCE/AHS/ASC 41st Structures, Structural Dynamics, and Materials Conference. No. 2000-1697, April 2000.

${ }^{13}$ Piatak, D. J. and Cleckner, C. S., "A New Forced Oscillation Capability for the Transonic Dynamics Tunnel." 40th Aerospace Sciences Meeting and Exhibit, Reno, NV, Jan. 2002.

${ }^{14}$ Schuster, D. M. and Rausch, R. D., "Transonic Dynamics Tunnel Force and Pressure Data Acquired on the HSR Rigid Semispan Model," NASA CR 1999-209555, Sept. 1999.

${ }^{15}$ Kris, S. L., Biedron, R. T., and Rumsey, C. L., "CFL3D User's Manual Version 5.0," NASA-TM 1998-208444, NASA Langley Research Center, Hampton, VA, June 1998.

${ }^{16}$ Biedron, R. T., "CFL3D Version 6.0 Website," http://fmad-www.larc.nasa.gov/ biedron/cfl3dv6/cfl3dv6.html, 2000.

${ }^{17}$ Bartels, R. E. and Gatski, T. B., "Prediction of Transonic Vortex Flows Using Linear and Nonlinear Turbulent Eddy Viscosity Models," NASA-TM 2000-210282, May 2000. 